\title{
ANTECEDENTES DE DESARROLLO Y POTENCIAL PRODUCTIVO DE VARIEDADES HIBRIDAS ENTRE Eucalyptus nitens y Eucalyptus globulus EN CHILE EXPERIENCIA CMPC ${ }^{8}$
}

\author{
Medina, Alex ${ }^{9}$; Emhart, Verónica ${ }^{9}$; Navarrete, Ricardo ${ }^{10}$; \\ Rothen, Berta ${ }^{9}$; Labra, Marcela ${ }^{9}$ y Velilla, Edgardo ${ }^{9}$
}

\section{RESUMEN}

El programa de híbridos de CMPC comenzó a inicios de la década del 90 del siglo pasado y se ha orientado desde entonces a desarrollar clones de variedades híbridas que por una parte maximicen las ganancias en rendimiento (ADT pulpa/ha) y calidad de fibra, y que por otra permitan alcanzar una mejor adaptabilidad y homogeneidad de plantaciones en cada condición de sitio de CMPC.

La estrategia Híbrida de CMPC se ha basado en el desarrollo de poblaciones sintéticas teniendo como material parental principal, genotipos selectos de Eucalyptus nitens y Eucalyptus globulus, más una batería de especies complementarias. Todo ello ha permitido generar en los últimos 10 años más de 150 mil progenies híbridas y testear en ensayos clonales más de 3.500 clones.

En el presente trabajo se evalúan y discuten los resultados de las etapas de cruzamiento y calidad de la semilla de tres años de cruzas de las variedades híbridas $\mathrm{F} 1$ entre $E$. nitens y $E$. globulus y sus retrocruzas y se presentan también los resultados de crecimiento a los 4 años de ensayos clonales en tres condiciones de sitios, para las mismas variedades híbridas.

Con la presentación de estos resultados se busca entregar antecedentes referenciales altamente valiosos y elementos de discusión asociadas a la selección de especies y variedades híbridas de alto potencial de productividad en sitios chilenos y de similares características de otras latitudes.

Palabras claves: Eucalyptus nitens, Eucalyptus globulus, Híbridos

\footnotetext{
8 Forestal Mininco SA. Compañía Manufacturera de Papeles y Cartones (CMPC)

9 CMPC, Chile, alex.medina@forestal.cmpc.cl

${ }^{10}$ IMF, Ricardo.navarrete@imf.cl
} 


\section{SUMMARY}

The CMPC hybrid program started at the 90s decade and since then it has been oriented to develop hybrid varieties that will maximize pulp yield (ADT /ha) and pulp quality, and improve the adaptability and homogeneity of forest plantation at any CMPC site.

The hybrid strategy has been based on developing multiespecific hybrid populations with E. nitens and E. globulus as parental materials. Thus, in the last 10 years the CMPC hybrid program has generated more than 150 thousand hybrid progenies and planted around 3.500 clones on clonal test.

In this paper three years of accumulated data on crossing and seed quality are evaluated for F1 En x Egg hybrid varieties and its backcross. In addition the performance of clones at age 4 in three different soil type for the same hybrid varieties is also evaluated.

The results presented in this paper represent a valuable referential material to be used for companies and researcher in their breeding programs.

Key words: Eucalyptus nitens, Eucayptus globulus, Hybrids 


\section{INTRODUCCIÓN}

En programas de mejoramiento genético operativos la hibridación está siendo usada ampliamente en el desarrollo de clones que permitan superar el desempeño de los mejores materiales de especies puras, esto en rasgos de productividad, calidad de madera, resistencia a enfermedades o tolerancia a algún estrés ambiental. Entre los géneros con mayor desarrollo de materiales híbridos operativos se encuentra sin duda el género Populus donde prácticamente el $100 \%$ de las plantaciones productivas manejadas se basan en clones híbridos (Zamudio et al. 2008).

Para el género Pinus el desarrollo de clones híbridos es más reciente, sin embargo ya existen híbridos de $P$. elliottii $x \quad P$. caribaea var. Hondurensis, $P$. patula $\times P$. tecunumanii y $P$. patula $\times P$. oocarpa que están siendo usados operacionalmente por diversas empresas al Sur de África, en zonas específicas de Queensland, Australia, y en el norte de Argentina (CAMCORE, 2007).

Por su parte los programas de mejora genética del género Eucalyptus a nivel mundial y especialmente en países como Brasil, Sud África, Argentina y Chile, han tenido un cambio creciente hacia el desarrollo de clones híbridos. Esto ha llevado a una mayor oferta y superficie plantada de clones híbridos de alto desempeño, complementando la oferta de materiales de especies puras y en algunos casos, especialmente en Brasil, los clones híbridos están sosteniendo el $100 \%$ de programas de plantaciones en varias empresas forestales.

La hibridación entre especies forestales puede generarse en forma espontánea en bosques naturales y plantaciones o bien en forma asistida a través de programas de cruzamientos controlados. La hibridación natural permite aumentar la variación genética de una especie y generalmente se genera entre especies cercanas taxonómicamente y en contacto geográfico. Por su parte, la hibridación asistida como herramienta de generación de nuevas variedades genéticas es mucho más agresiva y rompe estas barreras taxonómicas y geográficas, es así que es común encontrar en la literatura cruzas híbridas entre especies de géneros distintos como Castanea $\mathrm{x}$ Populus, Cupressus x Chamaecyparis y otras (Wright, 1962).

En el caso de género Eucalyptus, existe un gran número de especies, más de 800 especies y subespecies definidas morfológicamente, que cubren un amplio rango geográfico y de sitios en sus poblaciones originales. Muchas de estas especies presentan en forma individual características altamente deseables de adaptabilidad a sitios específicos y aplicaciones industriales. Así las cruzas interespecíficas posibilitan el desarrollo de híbridos capaces de manifestar ganancias simultaneas en los atributos individuales de ambos padres, esto sumado a la ocurrencia de heterosis, vigor hibrido donde las progenies hibridas presentan desempeños superiores al de sus progenitores, constituye las bases biológicas de la creación de individuos superiores a través de la hibridación.

Hasta el momento no existen bases teóricas consistentes que permitan predecir las formas de transmisión de características de padres a hijos en cruzas hibridas interespecíficas. Así, si bien existe una tendencia en las características de la madera a ser transmitida de forma proporcional desde padre y madre a su progenie, no existe un patrón de heredabilidad claro en rasgos de viabilidad de la semilla, crecimiento y adaptación (Volker, 2002).

Para muchos programas de mejora genética de eucalipto en el mundo, la hibridación interespecífica ha constituido la forma más rápida y eficiente de obtener altas ganancias genéticas en atributos de interés industrial y por su parte la clonación la forma más rápida y eficiente de incorporar estas ganancias a las plantaciones y procesos industriales.

En general el programa de híbridos de CMPC ha seguido un enfoque probabilístico donde la chance de encontrar clones híbridos superiores radica en la capacidad del programa de generar y testear una gran cantidad de genotipos a partir de las especies de interés ya 
reconocidas, además de la incorporación de especies de características complementarias.

El programa de híbridos de CMPC tiene como objetivo general producir variedades con mayor rendimiento, mejor adaptabilidad y homogeneidad que genotipos puros. Operativamente, el programa busca mantener 3 o más clones híbridos de ADT/ha/año igual o superior a los mejores genotipos puros de E. nitens y E. globulus, en cada condición de sitio.

Algunos objetivos específicos del programa de híbridos son aumentar la variabilidad genética intra-específica de $E$. globulus y $E$. nitens por medio de la hibridación entre éstas y otras especies de interés que aporten a los objetivos de mejora, generar nuevas variedades de genotipos de Eucalyptus adaptados a un rango más amplio de sitios que los definidos actualmente para $E$. globulus y E. nitens, y generar opciones estratégicas alternativas y de mayor plasticidad a las especies tradicionales, como resguardo frente a plagas, enfermedades y oportunidades de mercado.

El programa de híbridos CMPC ha puesto un gran énfasis en el desarrollo de clones de cruzas entre materiales elite de las especies $E$. nitens y $E$. globulus (Figura $\mathrm{N}^{\circ} 1$ ). Con esta variedad se busca generar clones que combinen el alto crecimiento y tolerancia al frío de $E$. nitens con la calidad de madera y enraizamiento de E. globulus, logrando variedades con un desempeño superior a los mejores genotipos puros de cada especie pura, por condición de sitio.

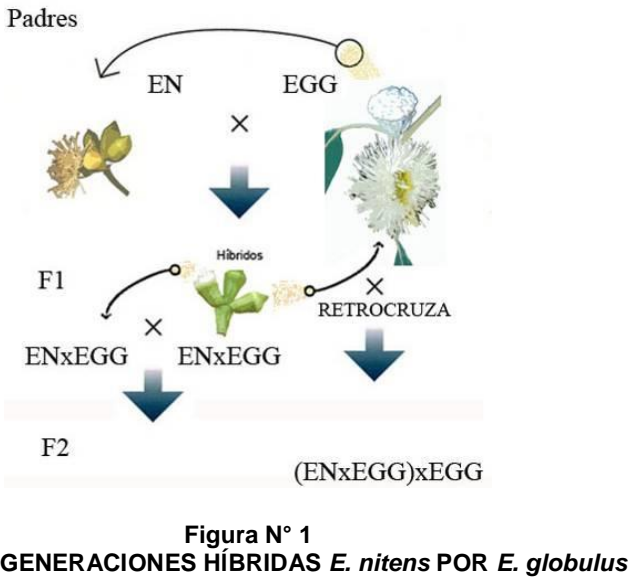

Los híbridos de primera generación entre dos especies son denominados $\mathrm{F} 1$, en esta etapa es prioritario lograr una gran cantidad de cruzas $\mathrm{F} 1 E$. nitens $\times E$. globulus, en adelante EnxEgg, de modo de aumentar la chance de encontrar clones de buen enraizamiento.

No obstante, no existe ninguna razón genética para creer que a través de híbridos $\mathrm{F} 1$, con contribuciones de 50 a $50 \%$ desde los progenitores, se lograrán las mejores ganancias. Así retrocruzas o cruzas introgresivas (cruzas de genotipos híbridos con uno de los padres originales) pueden ser más apropiadas cuando se busca dar mayor probabilidad de ocurrencia a características de uno de los padres.

Este es el caso de retrocruzas de (EnxEgg) xEgg, (75\% Egg y $25 \%$ En), donde se busca aumentar la capacidad de enraizamiento y dar mayor énfasis a las propiedades de la madera en un hibrido que ya presenta una alta tasa de crecimiento y tolerancia al frio. 
Como notación escrita, en una cruza híbrida $(A x B)$, A se refiere a la madre y $B$ al padre o donante de polen.

Una vez que se logran buenos híbridos $\mathrm{F} 1$ es posible pasar a la segunda generación de híbridos F2 al cruzar genotipos F1 no emparentados. Actualmente se han desarrollado cruzas F2 en ensayos de campo esperando ser evaluados, sin embargo los esfuerzos se han centrado en el desarrollo de híbridos $\mathrm{F} 1$ y retrocruzas.

El material para el desarrollo de estas cruzas F1 ha sido en su mayoría las mejores familias de $E$. nitens de procedencia Nueva Gales del Sur y Victoria Central de Australia como madres y polen de clones operacionales de Egg de buen enraizamiento y alto rendimiento pulpable.

\section{OBJETIVOS}

El presente documento tiene como objetivo entregar antecedentes generales del programa clonal híbrido de eucalipto de CMPC Chile y resultados relevantes de información capturada durante varias temporadas en parámetros de cruzas y crecimiento volumétrico de variedades híbridas entre E. nitens y E. globulus.

Estos antecedentes, si bien no son totalmente extrapolables a otras condiciones y materiales genéticos, constituyen información referencial útil para otros programas de cruzas híbridas y pone en perspectiva el alto potencial de productividad de clones híbridos de estas variedades en suelos y sitios de alta importancia para Chile y países con similitudes climáticas.

\section{MATERIAL Y MÉTODO}

\section{Cruzamientos Híbridos y Parámetros Seminales}

En el presente capítulo se entregarán resultados de parámetros de cruzamientos controlados, de la calidad de semilla y de la habilidad de enraizamiento de clones producidos en estas cruzas, para las variedades híbridas F1 EnxEgg, las retrocruzas (EnxEgg)xEgg y Eggx(EnxEgg).

Es importante destacar que los antecedentes aquí descritos corresponden a resultados obtenidos de los programas operacionales de cruza por lo tanto no existe un balance ni diseños experimental en variables de interés tales como clones usados como madres, fuentes de polen, número de aislaciones y otros aspectos.

Así, en cada variedad híbrida se realizaron un número variable de aislaciones por año, en que se utilizaron igualmente un número variables de familia. El resumen del número de familias y aislaciones realizadas se describe en el Cuadro $\mathrm{N}^{\circ} 1$.

Los cruzamientos controlados de todas las variedades hibridas y $E$. nitens fueron realizados bajo el método tradicional de aislación con bolsa.

Por su parte, en E. globulus las cruzas se realizaron bajo la técnica OSP (one stop pollination) en la que se realiza un retiro del anillo estaminal post antesis (Harbard et al., 2000).

Estas cruzas fueron realizadas en distintos huertos semilleros clonales de CMPC sobre madres seleccionadas por sus atributos de productividad floral y además ranking en volumen y densidad para las madres de E. nitens y E. globulus. 
Cuadro $\mathrm{N}^{\circ} 1$

CRUZAS HIBRIDAS POR VARIEDAD Y POR AÑOS DE CRUZAMIENTO

\begin{tabular}{|c|c|c|c|c|c|c|c|c|}
\hline \multirow{2}{*}{ Especie* } & \multirow{2}{*}{ Item } & \multicolumn{6}{|c|}{ Años } & \multirow{2}{*}{ Total } \\
\hline & & 2006 & 2007 & 2008 & 2009 & 2010 & 2011 & \\
\hline \multirow[b]{2}{*}{ (EnxEgg)xEgg } & Familias & 8 & 4 & 19 & 12 & 34 & 1 & 78 \\
\hline & \begin{tabular}{|l|} 
Flores \\
aisladas
\end{tabular} & 229 & 173 & 732 & 434 & 1.270 & 54 & 2.893 \\
\hline \multirow[b]{2}{*}{ EgbxEgg } & Familias & & 10 & & 4 & 10 & 23 & 47 \\
\hline & $\begin{array}{l}\text { Flores } \\
\text { aisladas }\end{array}$ & & 466 & & 83 & 500 & 1.259 & 2.308 \\
\hline \multirow[b]{2}{*}{ Eggx(EnxEgg) } & Familias & 8 & 2 & & & & & 10 \\
\hline & $\begin{array}{l}\text { Flores } \\
\text { aisladas }\end{array}$ & 424 & 66 & & & & & 490 \\
\hline \multirow[b]{2}{*}{ EnxEgg } & Familias & 20 & 51 & 12 & 27 & 15 & & 125 \\
\hline & \begin{tabular}{|l|} 
Flores \\
aisladas
\end{tabular} & 220 & 1.017 & 448 & 636 & 285 & & 2.606 \\
\hline \multirow[b]{2}{*}{ Total General } & Familias & 36 & 67 & 31 & 43 & 59 & 24 & 260 \\
\hline & $\begin{array}{l}\text { Flores } \\
\text { aisladas }\end{array}$ & 873 & 1.721 & 1.180 & 1.154 & 2.055 & 1.313 & 8.297 \\
\hline
\end{tabular}

* Egg: Eucalyptus globulus ssp globulus Egb: Eucalyptus globulus ssp bicostata En: Eucalyptus nitens

\section{Desempeño de Clones Híbridos en Ensayos Clonales}

Un eslabón de gran importancia en la estrategia de selección de clones híbridos de CMPC corresponde a los ensayos clonales T1. El objetivo del ensayo T1 consiste en hacer selección rápida de una gran cantidad de clones candidatos.

El ensayo T1 presenta un diseño de un árbol por parcela, 20 réplicas y es establecido en una serie de 4 a 8 sitios dependiendo de las variedades evaluadas.

Una vez evaluados los ensayos T1 y seleccionados los mejores clones, estos candidatos son propagados y llevados a un ensayo de validación de estructura de bloques y muy bien replicados.

Los ensayos evaluados en el presente trabajo corresponden a tres ensayos $\mathrm{T} 1$, los que fueron establecidos en suelos de arcilla con riego, arcilla secano y granítico de baja altura. La información detallada de estos ensayos se presenta en el Cuadro $\mathrm{N}^{\circ} 2$.

La medición normal de estos ensayos se realiza a los 2 y 4 años. Con la medición de los 4 años es posible evaluar, además del volumen, la densidad de la madera de los clones candidatos a través del instrumento Pilodyn.

El mencionado instrumento permite medir la resistencia a la penetración, variable medida en $\mathrm{mm}$ y altamente correlacionada con la densidad básica de la madera.

Si bien el Pilodyn no entrega la densidad real, su alta correlación con la variable permite hacer un ranking de los clones en función de la densidad. 
Cuadro $\mathrm{N}^{\circ} 2$

INFORMACION GENERAL DE ENSAYOS CLONALES EVALUADOS

\begin{tabular}{|l|c|c|c|}
\hline Item & Código 267 & Código 267 & Código 77 \\
\hline Año plantación & 2006 & 2006 & 2006 \\
Tipo suelo & Arcilla con Riego & Arcilla Secano & Granítico \\
Altitud (msnm) & 200 & 220 & 100 \\
Comuna & Mulchén & Mulchén & Nacimiento \\
Predio & Alicahue & Casas Quemadas & Santa Adriana \\
Precipitación (mm/año) & $1200-1500$ & $1200-1500$ & $1200-1500$ \\
Diseño & STP & STP & STP \\
Repeticiones & 20 & 20 & 20 \\
Tratamientos & 47 & 233 & 123 \\
Controles & 12 & 12 & 13 \\
Arboles ensayo & 1680 & 5280 & 3200 \\
Espaciamiento (m) & $2 \times 3,5$ & $2 \times 3,5$ & $2 \times 3,5$ \\
Superficie (ha) & 1,2 & 3,7 & 2,2 \\
Fecha plantación & 17 Nov. & 24 Oct. & 20 Oct. \\
Fertilización NPK (g/planta) & 150 & 150 & 150 \\
Preparación suelo & Casilla manual & Casilla manual & Casilla manual \\
\hline
\end{tabular}

Se presentan y discuten los resultados de la medición a los 4 años de volumen y Pilodyn para las variedades En, Egg, EnxEgg y $(E n x E g g) x$ Egg. El volumen individual se obtuvo a través de dos funciones de volumen desarrolladas por la empresa. La función de volumen de Egg fue usada para cubicar las variedades Egg y la retrocruza, mientras que la función de En fue utilizada en la cubicación de En y el Híbrido F1.

Con los datos de volumen y Pilodyn se generó una variable que se denominó Índice de Fibra (IF). Esta se calcula a través del producto del volumen por el inverso del Pilodyn. Este índice representa un indicador de la productividad de fibra de cada clon y permite realizar un ranking de alta precisión y bajo costo.

Finalmente tanto el volumen como el índice de fibra se presentan en términos de ganancias relativas porcentuales respecto a la media de cada réplica. Así, esta transformación de las variables Vol e IF permite estimar la superioridad promedio de cada clon respecto al promedio de todos los clones del ensayo.

\section{RESULTADOS Y DISCUSIÓN}

\section{Parámetros de Cruzas y Seminales}

\section{- $\quad$ Retención de Capsulas}

Los resultados de retención o set de capsulas, expresados como el número de capsulas cosechadas dividido por el número total de flores polinizadas, se presentan en la Figura $\mathrm{N}^{\circ} 2$, y los resultados del test de comparación de medias entre especies para las variables retención de cápsulas se presentan en el Cuadro $\mathrm{N}^{\circ} 3$. 


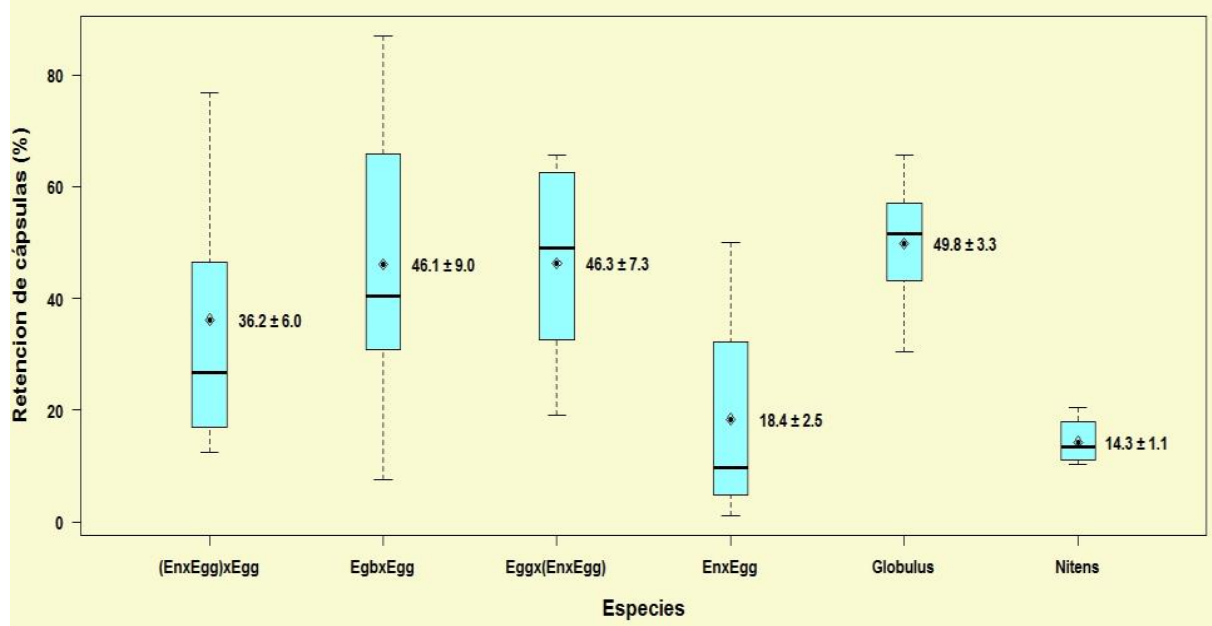

Boxplot es construido considerando la variación a través de las madres cruzadas en las distintas temporadas de cruzas

Figura $N^{\circ} 2$

RETENCIÓN DE CAPSULAS PARA DISTINTAS VARIEDADES HIBRIDAS Y PURAS DE EUCALIPTO

\section{Cuadro $\mathrm{N}^{\circ} 3$ \\ RESULTADOS DE TEST DE MEDIA PARA EL EFECTO ESPECIES, PARA LOS RASGOS RETENCION DE CAPSULAS, SEMILLAS POR CAPSULA Y CAPACIDAD GERMINATIVA}

\begin{tabular}{|l|c|c|c|}
\hline Especie & $\begin{array}{c}\text { Retención de Cápsula } \\
(\%)\end{array}$ & $\begin{array}{c}\text { Semillas/Cápsula } \\
\left(\mathbf{N}^{\circ}\right)\end{array}$ & $\begin{array}{c}\text { Capacidad Germinativa } \\
(\%)\end{array}$ \\
\hline Egg & $49.8 \mathrm{a}$ & $30.5 \mathrm{a}$ & $79.7 \mathrm{a}$ \\
\hline (EnxEgg)xEgg & $36.2 \mathrm{a}$ & $4.0 \mathrm{c}$ & $51.7 \mathrm{~b}$ \\
\hline EgbxEgg & $46.1 \mathrm{a}$ & $4.2 \mathrm{c}$ & $49.2 \mathrm{~b}$ \\
\hline Eggx(EnxEgg) & $46.3 \mathrm{a}$ & $15.5 \mathrm{~b}$ & $59.5 \mathrm{~b}$ \\
\hline EnxEgg & $18.4 \mathrm{~b}$ & $1.6 \mathrm{c}$ & $32.5 \mathrm{c}$ \\
\hline En & $14.3 \mathrm{~b}$ & $2.5 \mathrm{c}$ & $60.0 \mathrm{~b}$ \\
\hline
\end{tabular}

(Test de media $\mathrm{p}=0,05)$

De los resultados obtenidos se desprende que la variedad o especie con mayor retención de capsulas fue E. globulus, con un $49,8 \%$, seguida muy de cerca por las variedades Eggx(EnxEgg) y EgbxEgg, con un 46,3\% y 46,15\% de retención, respectivamente. La retrocruza (EnxEgg)x Egg obtuvo un $36,2 \%$ de retención mientras que la variedad F1 EnxEgg y E. nitens lograron una retención considerablemente menor, 18,4\% y $14,3 \%$, respectivamente.

Los resultados de test de media para la retención de capsulas confirman la existencia de diferencias significativas entre especies y permiten confirmar la existencia de dos grupos; grupo "a" compuesto por Egg y las variedades con mayor cercanía híbrida a esta especie Eggx(EnxEgg), EgbxEgg y (EnxEgg)x Egg y altos niveles de retención, y un segundo grupo "b" compuesto por En y el hibrido F1 EnxEgg. Las altas tasas de retención observadas en el grupo "a" son esperadas considerando las mayores similitudes anatómicas y compatibilidad de estructuras florales de la especie madre con el polen paternal. 
Por su parte, los resultados para En y su híbrido F1 son en general bajos y menores a algunos valores encontrados en la literatura. Al respecto, Tibbits (1989), en un estudio de polinización controlada de $E$. nitens, reporta valores de $45 \%$ de retención para cruzas controladas puras de E. nitens y $23 \%$ para cruzas EnxEgg. Volker (2002) reporta valores promedio de retención de capsulas de $61 \%$ para EggxEgg, 22\% para Egg autocruza, 23\% para EnxEgg, 29\% para EnxEn y $10 \%$ para En autocruza.

Son varios los autores que describen un efecto maternal en la taza de retención de capsulas al igual que en el número de semillas por capsula en eucalipto (Tibbits, 1989; Volker, 2002).

Así, es posible encontrar madres con buen set al ser cruzadas con una amplia variedad de pólenes y otras con mal set independiente del polen. Esta variación es evidente en el gráfico de caja donde se aprecia, en especial en las variedades híbridas, una alta variación entre madres. Teniendo esto en consideración y haciendo una revisión más detallada de la variación del set a través de las madres se observa que para la variedad $\mathrm{F} 1$, de las 45 madres cruzadas 7 presentaron un set mayor al $40 \%$.

Es importante mencionar que en la colección de cruzas híbridas evaluadas en este estudio no se hizo una selección de madres bajo sus méritos de cruzabilidad, sino más bien de acuerdo a su oferta de yemas florales en la temporada.

Actualmente en CMPC el programa de cruzas híbridas, especialmente en las variedades F1 y retrocruzas, considera parámetros de retención y cruzabilidad al momento de seleccionar las madres.

Lo anterior, junto a una buena selección y mantención del polen y un manejo de precisión del huerto de cruzas, ha permitido aumentar considerablemente los sets de cruzas y con ello mejorar la eficiencia y costos del programa de híbridos.

\section{Semillas por Capsula}

Los resultados de productividad de fruto, expresados como semillas por cápsula (sem/cap) se presentan en la Figura $\mathrm{N}^{\circ} 3$ y los resultados del test de comparación de medias entre especies para semillas por cápsula son lo presentados en el Cuadro $\mathrm{N}^{\circ} 3$.

En la Figura $\mathrm{N}^{\circ} 3$ es posible apreciar que existe una alta variación de este parámetro entre variedades híbridas. El test de media permite confirmar la significancia de estas diferencias y agrupar las 6 variedades en tres grupos; grupo "a" compuesto por Egg con alto número de semillas por cápsulas, grupo "b" compuesto por la variedad Eggx(EnxEgg) y finalmente un grupo "c" con menos de 4 sem/cap conformado por las variedades (EnxEgg)x Egg, EgbxEgg, EbxEgg y En.

Estos resultados armonizan con los entregados por Volker (2002) que reporta valores promedios de 38,4 sem/cap para cruzas EggxEgg; 3,7 sem/cap para EnxEgg y 6,4 sem/cap para cruzas de EnxEn. 


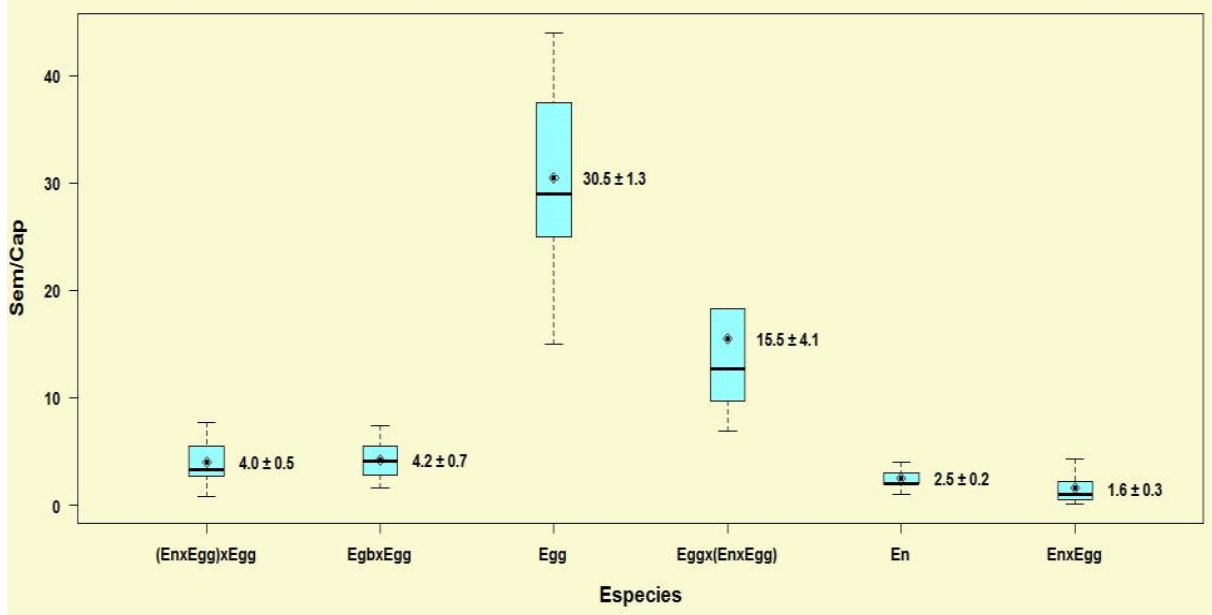

Boxplot es construido considerando la variación a través de las madres cruzadas en las distintas temporadas de cruzas.

\section{Figura $\mathrm{N}^{\circ} 3$}

\section{SEMILLAS POR CAPSULA PARA DISTINTAS VARIEDADES HIBRIDAS Y PURAS DE EUCALIPTO}

\section{- Capacidad Germinativa}

Los resultados denotan una importante variación en la capacidad germinativa, con diferencias significativas entre Egg, la variedad F1 EnxEgg y un tercer grupo compuesto por las variedades restantes.

Con la excepción de Egg que presentó un buena CG, todas las otras variedades presentaron valores moderados de CG, siendo la variedad F1 EnxEgg la de menor tasa de germinación, $32,5 \%$.

Es importante señalar que la germinación se realizó bajo dos protocolos de tratamientos pregerminativos desarrollados para Egg y En. Asi, la variedad F1 EnxEgg fue tratada bajo el protocolo de En mientras que las otras variedades híbridas fueron tratadas bajo el protocolo de Egg.

La diferencia entre ambos protocolos es básicamente el mayor tiempo de remojo de la semilla de En (48 horas) en una solución de con ácido giberélico a 300 ppm.

Los resultados de la capacidad germinativa (CG) expresada como el valor porcentual de la razón entre el número de semillas germinadas partido por el total de semillas viables sembradas se presentan en la Figura $\mathrm{N}^{\circ} 4$ y los resultados del test de comparación de medias entre especies para el rasgo capacidad germinativa son los presentados en el Cuadro $\mathrm{N}^{\circ} 3$. 


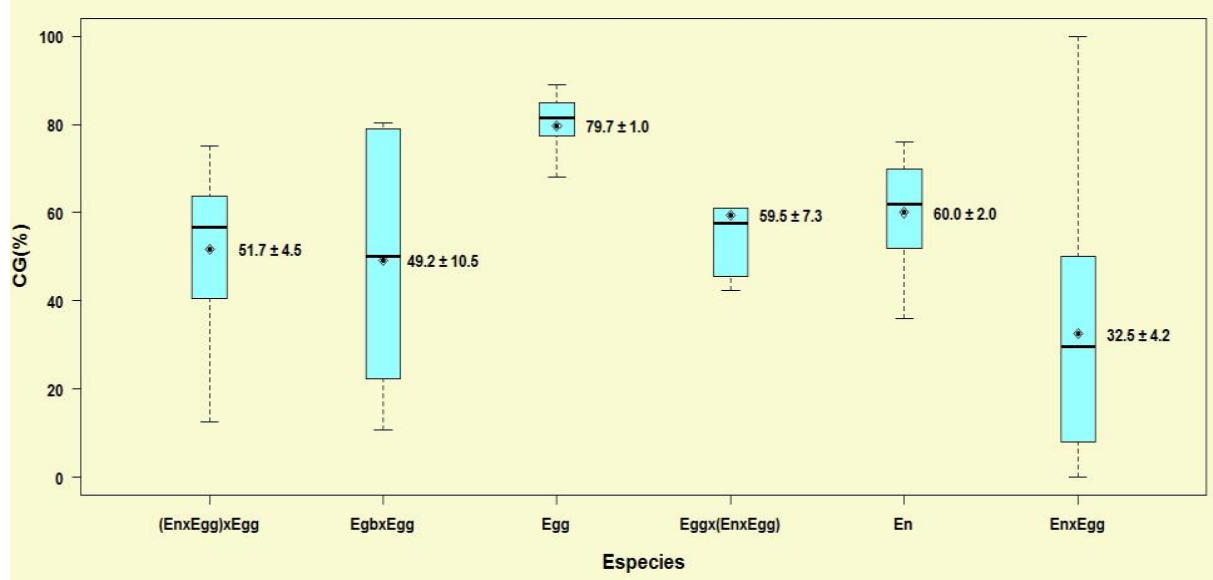

Boxplot es construido considerando la variación a través de las madres en las distintas temporadas de cruzas

\section{Figura $N^{\circ} 4$}

CAPACIDAD GERMINATIVA PARA DISTINTAS VARIEDADES HIBRIDAS Y PURAS DE EUCALIPTO

\section{Desempeño de Clones Híbridos en Ensayos Clonales}

\section{- $\quad$ Resultados para Arcilla con Riego}

Los resultados del volumen e índice de fibra se presentan en términos de ganancias relativas porcentuales del volumen (GR-Vol) y del índice de fibra (GR-IF) ordenados de mayor a menor en función del ranking de la GR-Vol (Figura $N^{\circ} 5$ ).

Las barras de la Figura $N^{\circ} 5$ han sido coloreadas según la variedad del tipo de material, donde Clon-F1 (azul) representan todos los clones candidatos de la variedad EnxEgg. Por su parte, los controles también han sido agrupados según su tipo. Clon-En (negro) corresponde a los genotipos control de En propagados por semilla. Ctrol-Hib OP (rojo) en tanto identifica a los controles híbridos que se usan operacionalmente. Esta nomenclatura es la misma en los gráficos presentados para los tres ensayos evaluados.

Es posible observar que alrededor del $50 \%$ de los genotipos evaluados presentaron una GR-Vol positiva. También se aprecia que existen 5 clones (10\% del total) de la variedad $\mathrm{F} 1$ que presentan una GR-Vol superior al $50 \%$ y están por encima del mejor control, en este caso un control de En.

Por otra parte, a través de la curva de GR-IF se observa que tanto los mejores y peores clones en volumen fueron y prácticamente en el mismo orden, los mejores y peores del ranking por su índice de fibra, lo que refleja una alta correlación de este índice con los valores extremos de volumen.

Por el contrario se observa una menor correspondencia entre el ranking de las dos variables en los genotipos con GR-Vol cercanos a cero. Un ejemplo claro de esto se presenta en el único clon de Egg (barra color verde), el cual presenta una GR-Vol del 13\% y una GR-IF del $48 \%$.

Más específicamente, la evaluación de este ensayo indica que de los 40 clones evaluados 8 (40\%) presentaron una GR-Vol y un GR-IF mayor al $40 \%$ y todos son de la variedad 
F1. Se aprecia también que 5 de estos clones tuvieron un desempeño mayor al mejor control, lo que otorga una gran potencial de selección de clones para ser llevados al ensayo de validación.

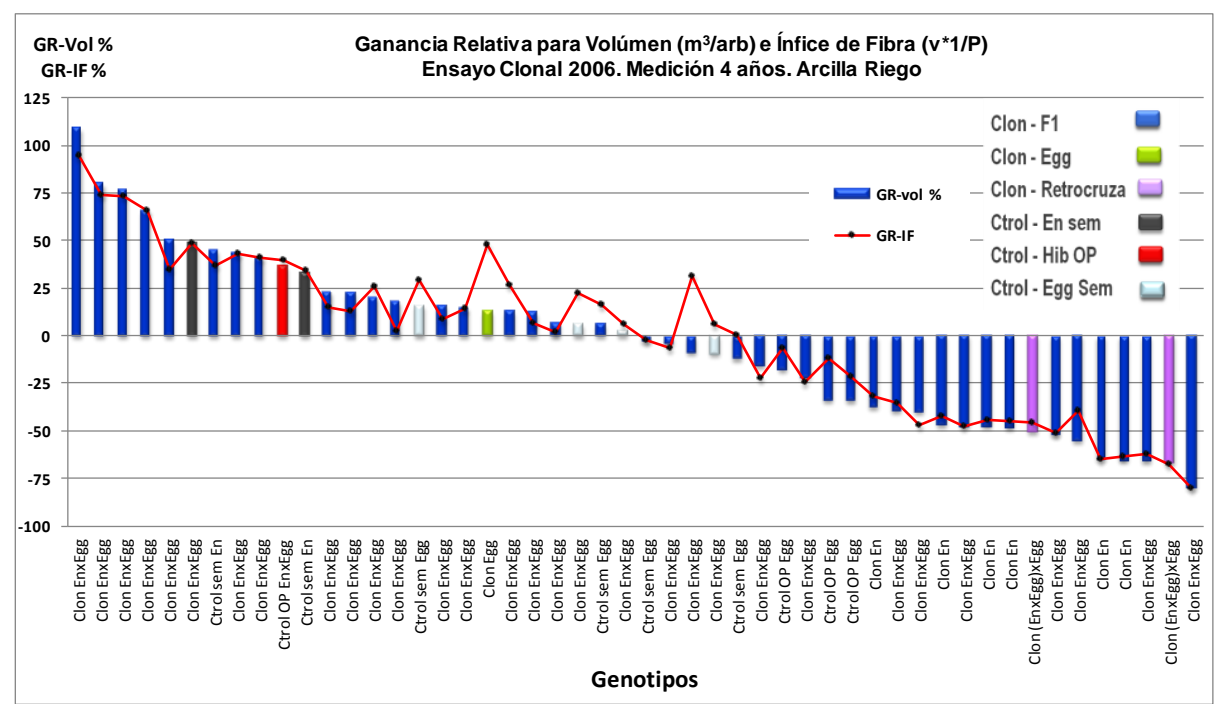

\section{Figura $\mathrm{N}^{\circ} 5$ \\ GANANCIA RELATIVA PARA VOLUMEN E INDICE DE FIBRA PARA LOS MEJORES 50 GENOTIPOS DEL RANKING POR VOLUMEN. ENSAYO ARCILLA RIEGO}

\section{- $\quad$ Resultados para Arcilla sin Riego}

Los resultados para el ensayo establecido en un suelo de arcilloso sin riego se observan en la Figura $\mathrm{N}^{\circ} 6$.

De los 245 genotipos presentes en este ensayo, solo 137 fueron medidos con Pilodyn, de ellos 11 corresponden a controles y los restantes 126 al mejor $54 \%$ de clones en su ranking volumétrico. Los análisis de GR-Vol y GR-IF se realizaron en este sub set de 137 genotipos.

Del análisis de los resultados se desprende que 61 (48\%) clones candidatos presentaron GR-Vol positivas. La gran amplitud de las GR-Vol (173\% a -70\%) denota una alta variabilidad del volumen entre clones.

Del análisis de la Figura $N^{\circ} 6$ se puede rescatar que el mejor control corresponde a un genotipo de Egg de semilla con un $108 \%$ de GR-Vol y un $98 \%$ en GR-IF lo cual es esperable ya que en arcilla secano Egg tiene un buen desempeño general.

En términos de clones candidatos, existen 4 clones EnxEgg que superan al mejor control seguidos por un clon candidato de Egg el que presenta una alta GR-IF.

Se puede indicar también que existen 36 genotipos con GR-Vol mayor al $50 \%$, de ellos 28 corresponden a clones candidatos donde 17 corresponden a clones F1, 10 a Egg y uno a retrocruza. 
Estos 28 candidatos ( $12 \%$ del total presente en el ensayo) constituyen materiales de alto potencial y califican para ser llevados a la serie de ensayos de validación.

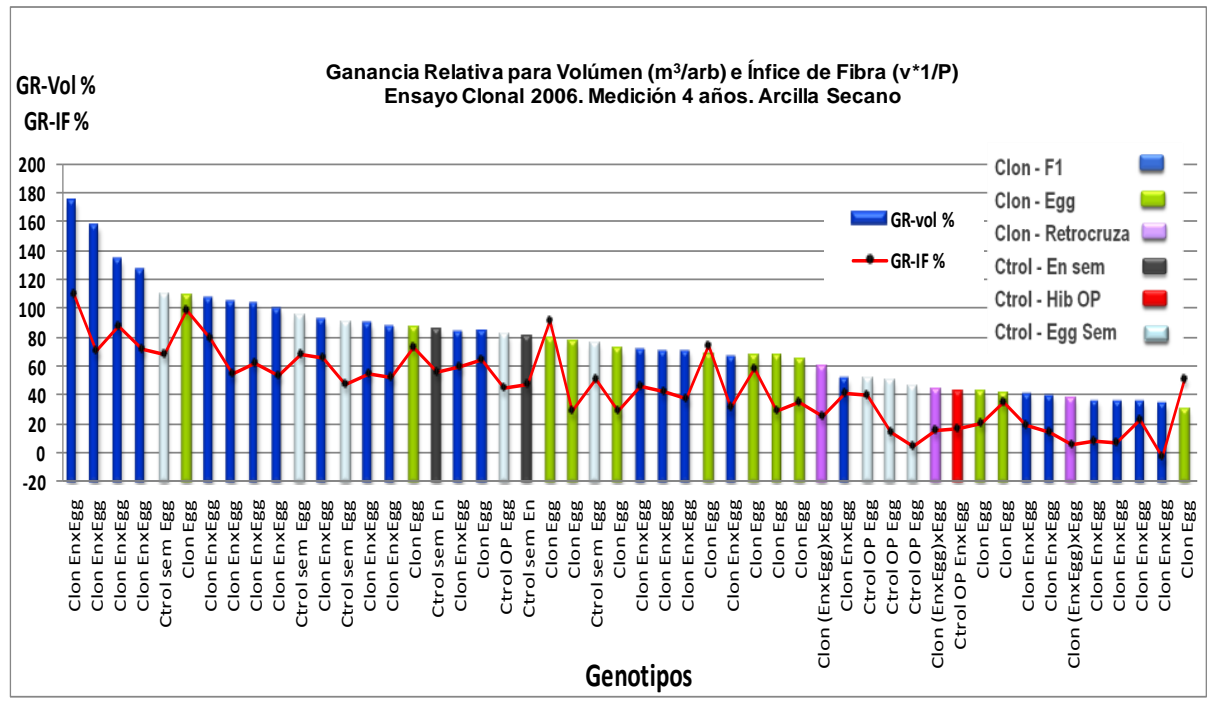

Figura $N^{\circ} 6$

GANANCIA RELATIVA PARA VOLUMEN E INDICE DE FIBRA PARA LOS MEJORES 50 GENOTIPOS DEL RANKING POR VOLUMEN. ENSAYO ARCILLA SECANO

\section{- Resultados para Suelo Granítico}

De los 136 genotipos presentes en este ensayo, solo 110 fueron medidos con Pilodyn y conforman el subset de datos sobre el cual se realizó el análisis de GR-Vol y GR-IF aquí presentado. Figura $\mathrm{N}^{\circ} 7$.

Los resultados para el ensayo establecido en un suelo de granítico se observan en la

Al revisar los datos evaluados se puede observar que 47 genotipos (42\%) presentaron GR-Vol positiva.

El material control de mejor desempeño en este sitio fue En llegando a un $47 \%$ de GRVol y un $44 \%$ en GR-IF.

Respecto a los clones candidatos, de los 123 clones evaluados en el ensayo 19 presentaron una GR-Vol mayor al 40\%. De ellos, un clon corresponde a una retrocruza, un clon a Egg y 17 a la variedad EnxEgg.

Es interesante ver como la mayor densidad de este clon de Egg le otorga una mayor GRIF $(61 \%)$ respecto a muchos de los clones mejor posicionados en el ranking de GR-Vol. 


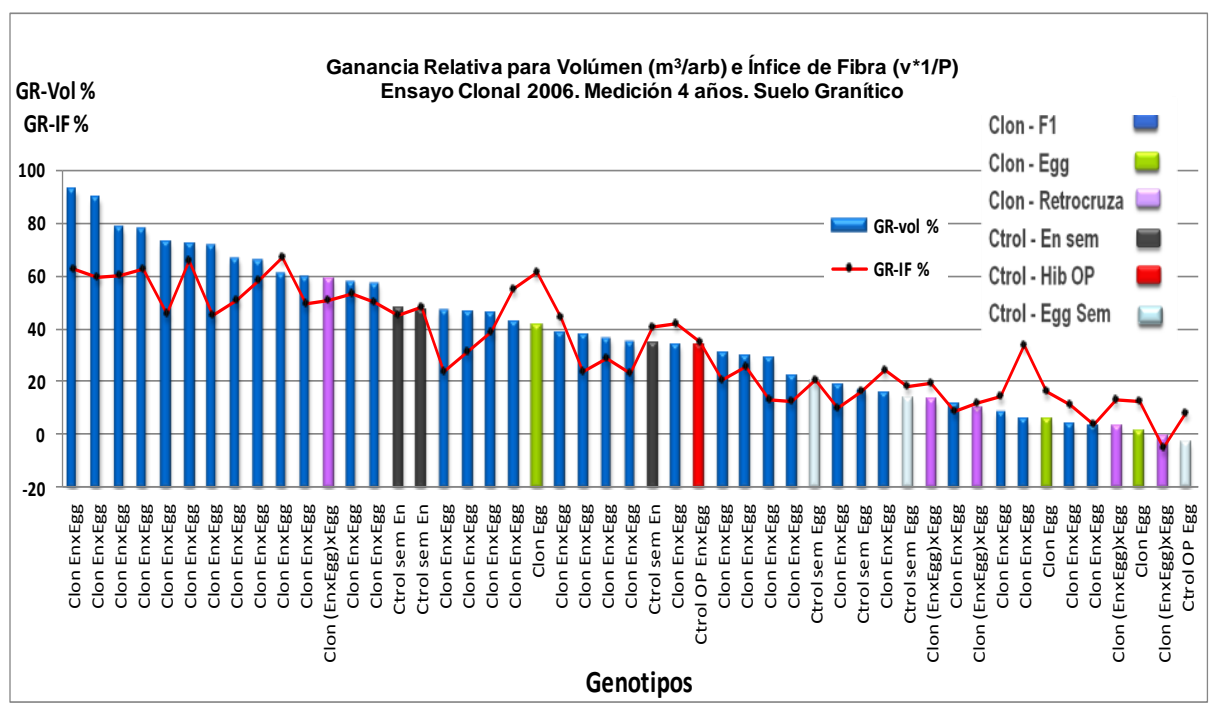

Figura $N^{\circ} 7$

GANANCIA RELATIVA PARA VOLUMEN E INDICE DE FIBRA PARA LOS MEJORES 50 GENOTIPOS DEL RANKING POR VOLUMEN. ENSAYO SUELO GRANÍTICO

\section{CONCLUSIONES}

La evaluación de la retención de cápsulas manifiesta la existencia de dos grupos de especies, uno compuesto por Egg y las variedades con mayor cercanía híbrida a esta especie Eggx(EnxEgg), EgbxEgg y (EnxEgg)x Egg los que manifiestan altos niveles de retención, y un segundo grupo compuesto por En y el hibrido F1 EnxEgg con menores tasas e retención.

Para la variable semillas por cápsula también se observaron variaciones significativas entre especies, siendo nuevamente Egg la especie con mejor desempeño con una media de 30,1 sem/cap, seguido por la variedad Eggx(EnxEgg) con 15,5 sem/cap. Las especies restantes presentaron valores menores a 4,2 sem/cap.

Para la capacidad germinativa los resultados denotan una variación significativa entre especies con un alto desempeño para Egg 80\% CG y moderado para el resto de las especies.

El registro y conocimiento a nivel de variedades, madres y familias de los atributos de retención de cápsulas, productividad del fruto (sem/cap) y capacidad germinativa, entre otras, es elemental y de alto impacto en la eficiencia y éxito de un programa de cruzas híbridas. En la medida que se logra un mejor manejo de estas variables se reducen los costos del programa, se acelera la evaluación de familias hibridas elite y se aumenta la probabilidad de seleccionar clones de alto desempeño.

La evaluación del desempeño de clones candidatos en ensayos T1 en tres condiciones de sitio reflejó una alta adaptabilidad y potencial de selección de clones de la variedad EnxEgg en las tres condiciones de sitio. Por su parte los clones de Egg también presentaron un excelente desempeño en arcilla sin riego. 


\section{REFERENCIAS}

CAMCORE, 2007. Boletín de Noticias CAMCORE para México y Centro América. 2007. Volumen 1, número 4. En: http://www.camcore.org/publications/documents/boletincamcore2007_4octubre.pdf

Harbard, J. L.; Griffin, R.; Espejo, J. E.; Centurion, C. and Russell, J., 2000. 'One stop pollination' a new technology developed by Shell Forestry technology unit. In: Dungey HS, Dieters MJ, Nikles DG (eds) Proceedings of QFRI/CRC-SPF symposium: hybrid breeding and genetics of forest trees. Department of Primary.

Tibbits, W. N., 1989. Controlled pollination studies with Shining Gum (Eucalyptus nitens (Deane and Maiden) Maiden). Forestry 62, 111-126

Volker, P. W., 2002. Quantitative genetics of Eucalyptus globulus, E.nitens and their $\mathrm{F}_{1}$ hybrid. PhD thesis, University of Tasmania.

Wright, J. W., 1962. Genetics of forest tree improvement. Rome. FAO Forestry and Forest Products Studies No. 16. 399 p.

Zamudio, A. F.; Baettig, P. R. y Guerra, G. F., 2008. Origen y futuro del cultivo del álamo en Chile. Universidad de Talca (Chile). Centro Tecnológico del Álamo. 
